\title{
Диффузионная динамика ридберговских состояний в поле излучения с непрерывным спектром
}

\author{
() А.С. Червинская, Д.Л. Дорофеев, Б.А. Зон \\ Воронежский государственный университет, \\ 394006 Воронеж, Россия \\ e-mail: dmitrii.dorofeev@gmail.com
}

Поступила в редакцию 29.07.2020 г.

В окончательной редакции 17.03.2021 г.

Принята к публикации 22.03.2021 г.

\begin{abstract}
Рассмотрены перераспределение заселенности ридберговских состояний и ионизация атома в результате многоступенчатых каскадных переходов между состояниями под действием излучения с непрерывным спектром. Рассмотрение производится как путем непосредственного численного решения кинетических уравнений для заселенности состояний, так и посредством диффузионного приближения на основе уравнения Фоккера-Планка. На примере атома натрия, на который действует излучение с прямоугольным спектром, показано хорошее согласие диффузионного приближения с численным решением кинетических уравнений. В рамках диффузионного приближения получены оценки для заселенности состояний, скорости ионизации и минимальной спектральной плотности излучения, необходимой для значительной ионизации.
\end{abstract}

Ключевые слова: диффузионная ионизация, ридберговские состояния.

DOI: $10.21883 /$ OS.2021.07.51070.207-20

\section{1. Введение}

Экспериментальное наблюдение многофотонной ионизации ридберговских состояний атома водорода СВЧ-излучением [1] (см. также [2]) стимулировало большое количество теоретических исследований, посвященных квантовой диффузии и классической теории возникновения и развития хаотического движения. Модель квантовой диффузии для указанного процесса была предложена в работе [3]. В этой модели внешнее поле считалось монохроматическим, что соответствовало условиям эксперимента [1]. Для появления в атомном спектре достаточного количества переходов с частотой, равной частоте воздействующего излучения, в работе [3] предполагалось значительное расщепление атомных уровней сильным полем. Дальнейший анализ вопроса [4] показал, что для этого необходимо поле с напряженностью порядка $10-20 \%$ от напряженности атомного поля на соответствующих возбужденных состояниях.

Суть механизма диффузионной ионизации состоит в том, что атом, находившийся в начальный момент времени в состоянии, прямая фотоионизация из которого невозможна или маловероятна, вследствие многоступенчатых каскадных переходов между уровнями под действием внешнего излучения приходит в какое-либо высоковозбужденное состояние, из которого он быстро ионизируется. Название данного механизма связано с тем, что многоступенчатые каскадные переходы эффективно описываются как процесс постепенной диффузии заселенности из начального состояния в состояния, всё более отличающиеся от него по энергии.
Классическое рассмотрение этой задачи, в частности хаотизация из-за переменного электромагнитного поля регулярных траекторий заряженной частицы в кулоновском поле, проводилось в большом числе работ. В качестве примера можно указать статьи [5-7]. Одними из первых обзорных статей, посвященных рассматриваемому вопросу, являются статьи $[8,9]$.

В настоящей работе рассматривается диффузионная ионизация под действием излучения с непрерывным спектром. В этом случае для реализации каскадных процессов не требуется сильное возмущение атомного спектра и соответственно эти процессы оказываются возможными при более низкой интенсивности излучения.

По сравнению с диффузионной ионизацией в поле монохроматического излучения для излучения с непрерывным спектром данный тип ионизации исследован значительно меньше. В работе Каулакиса [10] была теоретически рассмотрена диффузионная ионизация ридберговских состояний в поле чернотельного излучения, однако при этом из модели была полностью исключена прямая фотоионизация из начального состояния, идущая одновременно с диффузионной. В экспериментальной работе [11] было отмечено существенное влияние каскадных процессов на вероятность фотоионизации, однако систематического исследования этих процессов не проводилось. Следует отметить также публикации Бетерова и сотрудников, которые экспериментально измерили скорость ионизации ридберговских состояний атомов щелочных металлов под действием чернотельного излучения [12], а также провели соответствующие теоретические расчеты $[13,14]$, учитывая при этом кас- 
кадные процессы, но с длиной каскадов, не превышающей четыре ступени.

Сложность учета многоступенчатых каскадных процессов заключается в том, что с увеличением количества ступеней резко увеличивается количество состояний, которые необходимо включать в расчет. Решить эту проблему позволяет переход от дискретного представления процесса (в виде кинетических уравнений) к непрерывному представлению - диффузионному приближению на основе уравнения Фоккера-Планка. Аналогичный подход активно используется для расчета столкновительной ионизации (см., например, [15-17]), однако для фотоионизации данный подход менее разработан.

В настоящей работе проводится систематический учет многоступенчатых каскадных процессов в диффузионном приближении на основе уравнения Фоккера-Планка, что делает рассмотрение более наглядным и допускающим качественные оценки. Мы показываем, что диффузионный процесс перераспределения заселенности по состояниям может приводить к существенному изменению вероятности ионизации по сравнению с прямой фотоионизацией из начального состояния. В качестве модельного объекта выбран атом Na. Рассмотрена его фотоионизация под действием излучения с прямоугольным спектром. Вместе с тем полученные результаты несложно обобщить на случай, например, чернотельного излучения. Результаты, полученные с использованием диффузионного приближения, сравнены с расчетом непосредственно на основе системы кинетических уравнений для заселенностей состояний атома.

Bсе величины, если не указано иное, даны в атомных единицах.

\section{2. Диффузия по состояниям под действием излучения с непрерывным спектром}

Будем рассматривать поле малой интенсивности с непрерывным спектром. В этом случае можно пренебречь динамическим эффектом Штарка [3] и рассматривать эволюцию системы как последовательность переходов между уровнями с различными значениями главного и орбитального квантовых чисел. Будем считать, что в начальный момент времени электрон находится в некотором состоянии $\left(n_{0}, l_{0}\right)$, а последующие переходы и ионизация атома определяются системой кинетических уравнений:

$$
\begin{aligned}
\frac{d N_{n l}}{d t}= & \sum_{n^{\prime} l^{\prime}} V\left(n^{\prime} l^{\prime} \rightarrow n l\right) N_{n^{\prime} l^{\prime}} \\
& -N_{n l}\left(\sum_{n^{\prime} l^{\prime}} V\left(n l \rightarrow n^{\prime} l^{\prime}\right)+W_{i}(n l)\right),
\end{aligned}
$$

где $N_{n l}$ - заселенность состояния $(n l), V\left(n l \rightarrow n^{\prime} l^{\prime}\right)-$ вероятность перехода $n l \rightarrow n^{\prime} l^{\prime}$, равная сумме вероятно-

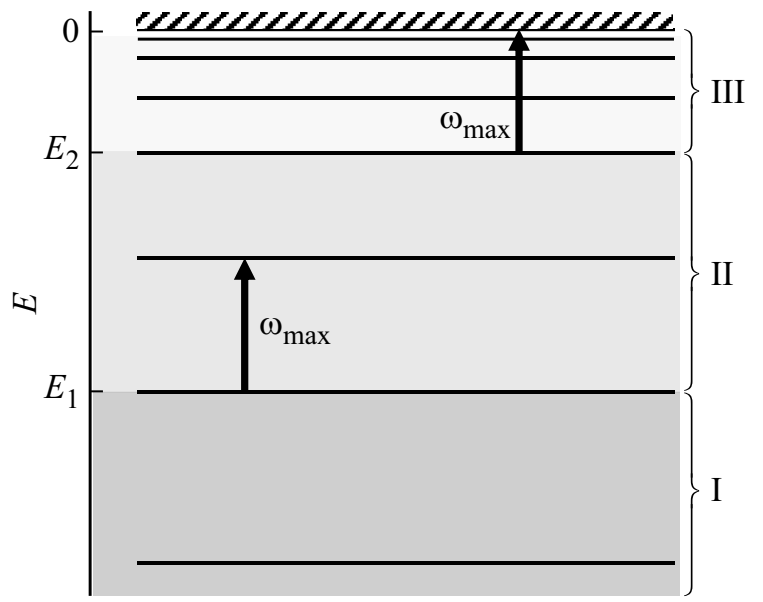

Pис. 1. Области в энергетическом спектре электрона: I невозможны никакие вынужденные переходы; II - возможны вынужденные связанно-связанные переходы, но не прямая фотоионизация; III - возможна прямая фотоионизация; $\omega_{\max }$ максимальная частота в спектре излучения, действующего на атом.

стей вынужденного и спонтанного переходов

$$
V\left(n l \rightarrow n^{\prime} l^{\prime}\right)=V_{\mathrm{ind}}\left(n l \rightarrow n^{\prime} l^{\prime}\right)+V_{\mathrm{sp}}\left(n l \rightarrow n^{\prime} l^{\prime}\right),
$$

$W_{i}(n l)$ - вероятность прямой фотоионизации электрона из состояния $(n l)$.

В данной работе мы ограничиваемся только случаем стохастического излучения, фазы спектральных компонент которого представляют равномерно распределенные случайные величины. Именно это позволяет описать воздействие излучения системой кинетических уравнений (1) и пренебречь интерференционными эффектами.

Далее будем считать, что спектральная плотность действующего на атом излучения сосредоточена между граничными значениями частоты $\omega_{\min }$ и $\omega_{\max }$. В этом случае можно разделить все спектральные уровни атома на три группы в зависимости от их энергии (рис. 1).

I. $E_{n l}<E_{1}$ : область, где частоты всех разрешенных переходов превосходят $\omega_{\max }$, соответственно, излучение не может индуцировать какие-либо переходы в данной области.

II. $E_{1}<E_{n l}<E_{2}=-\omega_{\max }$ : область, где излучение может индуцировать связанно-связанные переходы, но прямая фотоионизация невозможна.

III. $E_{2}<E_{n l}<0$ : область, где становится возможной прямая фотоионизация.

Значения главных квантовых чисел, соответствующие $E_{1}$ и $E_{2}$, обозначим $n_{1}$ и $n_{2}$.

Наибольший интерес для нас представляет область II, поэтому далее будем считать, что начальное состояние электрона $\left(n_{0}, l_{0}\right)$ находится в этой области.

Выясним, насколько быстро происходит переход в область III, где возможна прямая фотоионизация. Используя в качестве модельного объекта атом натрия, на который действует излучение с прямоугольным спектром 


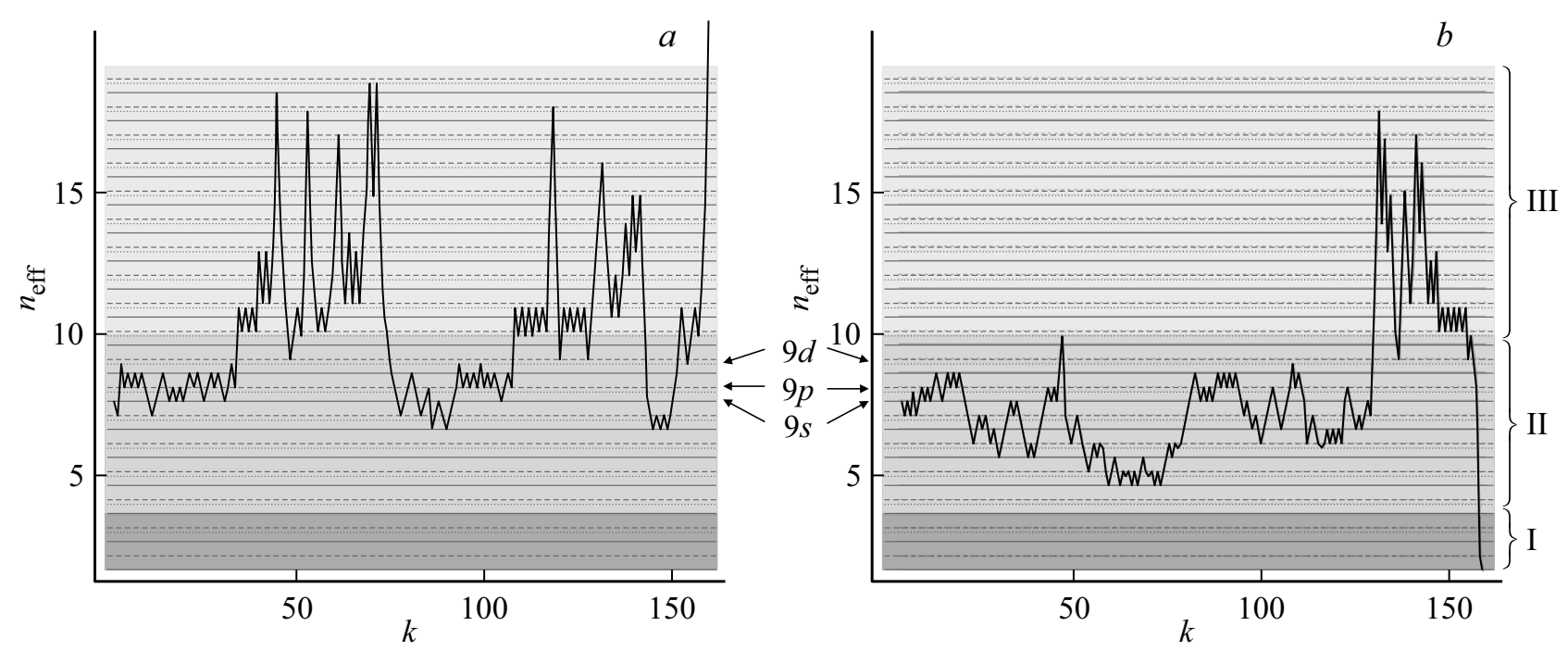

Рис. 2. Примеры блуждания электрона по энергетическим уровням в атоме Na из начального состояния $9 s$ под действием излучения с граничными частотами $\omega_{\min }=7 \cdot 10^{-4}, \omega_{\max }=5 \cdot 10^{-3}$ и энергетической спектральной плотностью излучения $\rho=4 \cdot 10^{-14}$. По оси абсцисс отложен номер перехода, т. е. порядковый номер ступени каскада $k$. По оси ординат отложено эффективное квантовое число $n_{\mathrm{eff}}=n-\mu$, где $\mu$ - квантовый дефект состояния. Горизонтальными линиями показаны состояния $s, p$ и $d$ (сплошные, штриховые и пунктирные линии соответственно). В результате блуждания, показанного на рисунке $a$, атом ионизируется, тогда как в случае $b$ атом в результате спонтанного перехода возвращается в основное состояние.

с граничными частотами $\omega_{\min }=7 \cdot 10^{-4}, \omega_{\max }=5 \cdot 10^{-3}$ и энергетической спектральной плотностью излучения $\rho=4 \cdot 10^{-14}$, рассмотрим процесс блуждания электрона по состояниям. На рис. 2 представлены результаты численного моделирования двух реализаций такого процесса: когда блуждание заканчивается ионизацией и когда блуждание заканчивается спонтанным переходом в основное состояние. В расчетах использовались формулы для матричных элементов связанно-связанных и связанно-свободных переходов в ридберговских атомах, приведенные в работах [18-22], и модельный потенциал для атома натрия, предложенный в [23]. Моделирование было выполнено в классической модели случайных блужданий с описанными выше вероятностями переходов. Как можно видеть, электрон совершает достаточно длительный путь по уровням, что связано с малостью вероятности ионизации и спонтанных переходов по отношению к вероятности вынужденных переходов в дискретном спектре.

Отметим также, что блуждания, представленные на рис. 2, происходят преимущественно между состояниями $s$ и $p$. Это связано с тем, что при выбранных параметрах излучения в рассматриваемых областях спектра натрия вероятности переходов между этими состояниями значительно выше, чем между другими. Это позволяет оставить в (1) только состояния $s$ и $p$. Также мы перейдем в (1) к суммарным заселенностям

$$
N(n)=N(n, s)+N(n, p),
$$

складывая уравнения для $N(n, s)$ и $N(n, p)$ и усредняя коэффициенты в правых частях уравнений.

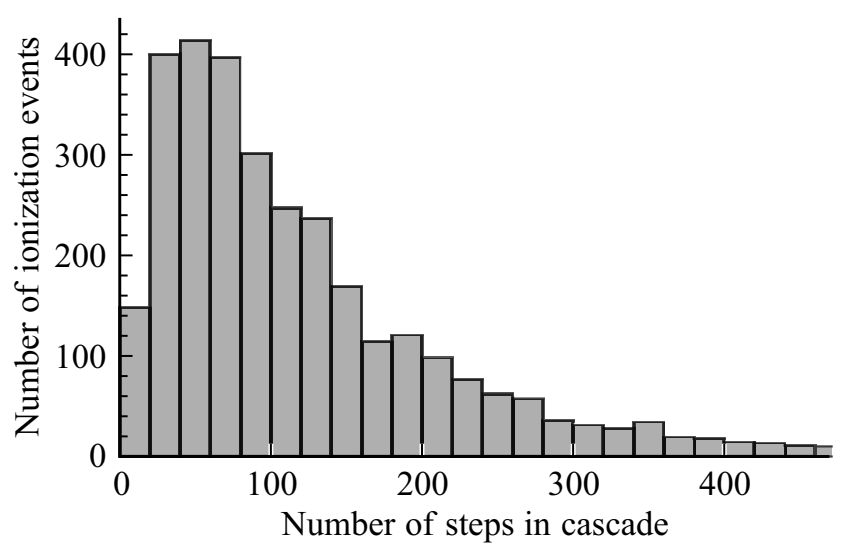

Рис. 3. Количество событий ионизации с заданным числом ступеней в каскаде. Начальное состояние атома и параметры излучения такие же, как на рис. 2. Общее количество рассмотренных событий ионизации: 10000 . Можно видеть, что наиболее часты каскады с количеством ступеней порядка 40-60.

Оценим теперь, какой вклад вносят многоступенчатые каскады в суммарную вероятность ионизации. На рис. 3 показано распределение событий ионизации по длине приводящих к ним каскадов. Видно, что преобладают каскады длиной 40-60 ступеней, т. е. достаточно длинные. Такие длительные случайные блуждания допускают эффективное описание этого процесса как процесса диффузии по состояниям, определяемого уравнением Фоккера-Планка $[3,10,15]$. 


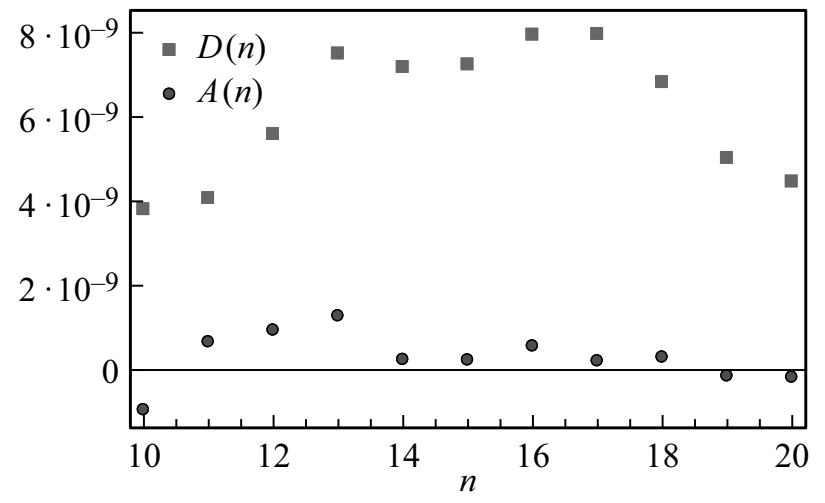

Рис. 4. Коэффициенты $D(n), A(n)$ для переходов в атоме Na. Начальное состояние и параметры излучения такие же как на рис. 2.

Следуя [16,24], перейдем от (1) к уравнению ФоккераПланка:

$$
\frac{\partial N}{\partial t}=\frac{\partial}{\partial n}(A N)+\frac{\partial^{2}}{\partial n^{2}}(D N)-\left(W_{i}+W_{q}\right) N
$$

где

$$
\begin{gathered}
A(n)=\frac{1}{2} \sum_{n^{\prime}>n_{1}}\left[V\left(n s \rightarrow n^{\prime} p\right)+V\left(n p \rightarrow n^{\prime} s\right)\right]\left(n^{\prime}-n\right) \\
D(n)=\frac{1}{4} \sum_{n^{\prime}>n_{1}}\left[V\left(n s \rightarrow n^{\prime} p\right)+V\left(n p \rightarrow n^{\prime} s\right)\right]\left(n^{\prime}-n\right)^{2}, \\
W_{i}(n)=\frac{1}{2}\left(W_{i}(n s)+W_{i}(n p)\right) \\
W_{q}(n)=\frac{1}{2} \sum_{n^{\prime} \leq n_{1}} V\left(n s \rightarrow n^{\prime} p\right)+V\left(n p \rightarrow n^{\prime} s\right)
\end{gathered}
$$

Можно видеть, что уравнение (2) представляет из себя уравнение диффузии с дополнительными слагаемыми, соответствующими утечке заселенности в область I и в непрерывный спектр.

Расчет коэффициентов (3), (4), результаты которого приведены на рис. 4, показывает, что в широком диапазоне характеристик излучения и в широком интервале значений $n$ справедливы допущения: (i) $D \gg A$; (ii) $D$ слабо зависит от $n$. С учетом этих допущений в области II уравнение (2) принимает следующий вид:

$$
\frac{\partial N_{\mathrm{II}}}{\partial t}=D \frac{\partial^{2} N_{\mathrm{II}}}{\partial n^{2}}-W_{q} N_{\mathrm{II}} .
$$

К уравнению (5) необходимо добавить граничное условие:

$$
\left.\frac{d N_{\mathrm{II}}}{d n}\right|_{n=n_{1}}=0,
$$

означающее нулевой поток заселенности через границу $n=n_{1}$, поскольку за счет вынужденных переходов электрон не может перейти из области II в область I.
В области III уравнение (2) выглядит так:

$$
\frac{\partial N_{\mathrm{III}}}{\partial t}=D \frac{\partial^{2} N_{\mathrm{III}}}{\partial n^{2}}-\left(W_{i}+W_{q}\right) N_{\mathrm{III}} .
$$

При этом на границе $n=n_{2}$ должны выполняться условия непрерывности:

$$
N_{\text {II }}=N_{\text {III }}, \quad \frac{d N_{\text {II }}}{d n}=\frac{d N_{\text {III }}}{d n} .
$$

Уравнения (5) и (7) вместе с граничным условием (6) и условиями непрерывности (8) допускают аналитическое решение, однако оно оказывается слишком громоздким и не позволяет сделать какие-либо качественные оценки. Поэтому мы принимаем дальнейшие упрощения: пренебрегая граничными условиями (8), воспользуемся для области II известным решением для полубесконечного стержня:

$$
\begin{aligned}
N_{\text {II }}= & \frac{\exp \left(-w_{q} t\right)}{2 \sqrt{\pi D t}}\left(\exp \left(-\frac{\left(n-n_{0}\right)^{2}}{4 D t}\right)\right. \\
& \left.+\exp \left(-\frac{\left(n-2 n_{1}+n_{0}\right)^{2}}{4 D t}\right)\right) .
\end{aligned}
$$

При этом мы принимаем, что вероятность перехода в область I имеет приближенно постоянное значение: $W_{q}(n) \approx w_{q}=$ const.

Далее мы пользуемся решением (9) для того, чтобы определить поток на границе $n=n_{2}$ :

$$
\begin{aligned}
& \left.\frac{\partial N_{\mathrm{II}}}{\partial n}\right|_{n=n_{2}}=\frac{\exp \left(-w_{q} t\right)}{4 \sqrt{\pi D^{3} t^{3}}}\left[\left(n_{2}-n_{0}\right) \exp \left(-\frac{\left(n_{2}-n_{0}\right)^{2}}{4 D t}\right)\right. \\
& \left.+\left(n_{2}-2 n_{1}+n_{0}\right) \exp \left(-\frac{\left(n_{2}-2 n_{1}+n_{0}\right)^{2}}{4 D t}\right)\right] .
\end{aligned}
$$

Подставив теперь (10) в (8), мы можем решить уравнение (7). На рис. 5 представлена динамика перераспределения заселенности по уровням, полученная численно из уравнения (1) (точки) и рассчитанная на основе диффузионного приближения (5)-(10) (сплошные линии). Как видно, диффузионное приближение очень хорошо согласуется с численным расчетом. Этот факт можно также рассматривать как обоснование сделанных выше приближений.

Теперь перейдем к рассмотрению полной вероятности ионизации в единицу времени

$$
\Omega_{i}=\int_{n_{2}}^{\infty} W_{i}(n) N_{\mathrm{III}}(n) d n .
$$

При этом сделаем еще одно приближение - примем, что в области III скорость прямой фотоионизации имеет примерно равное значение для всех состояний $W_{i}(n) \approx w_{i}=$ const. Тогда

$$
\Omega_{i}=w_{i} P, \quad P=\int_{n_{2}}^{\infty} N_{\mathrm{III}}(n) d n .
$$



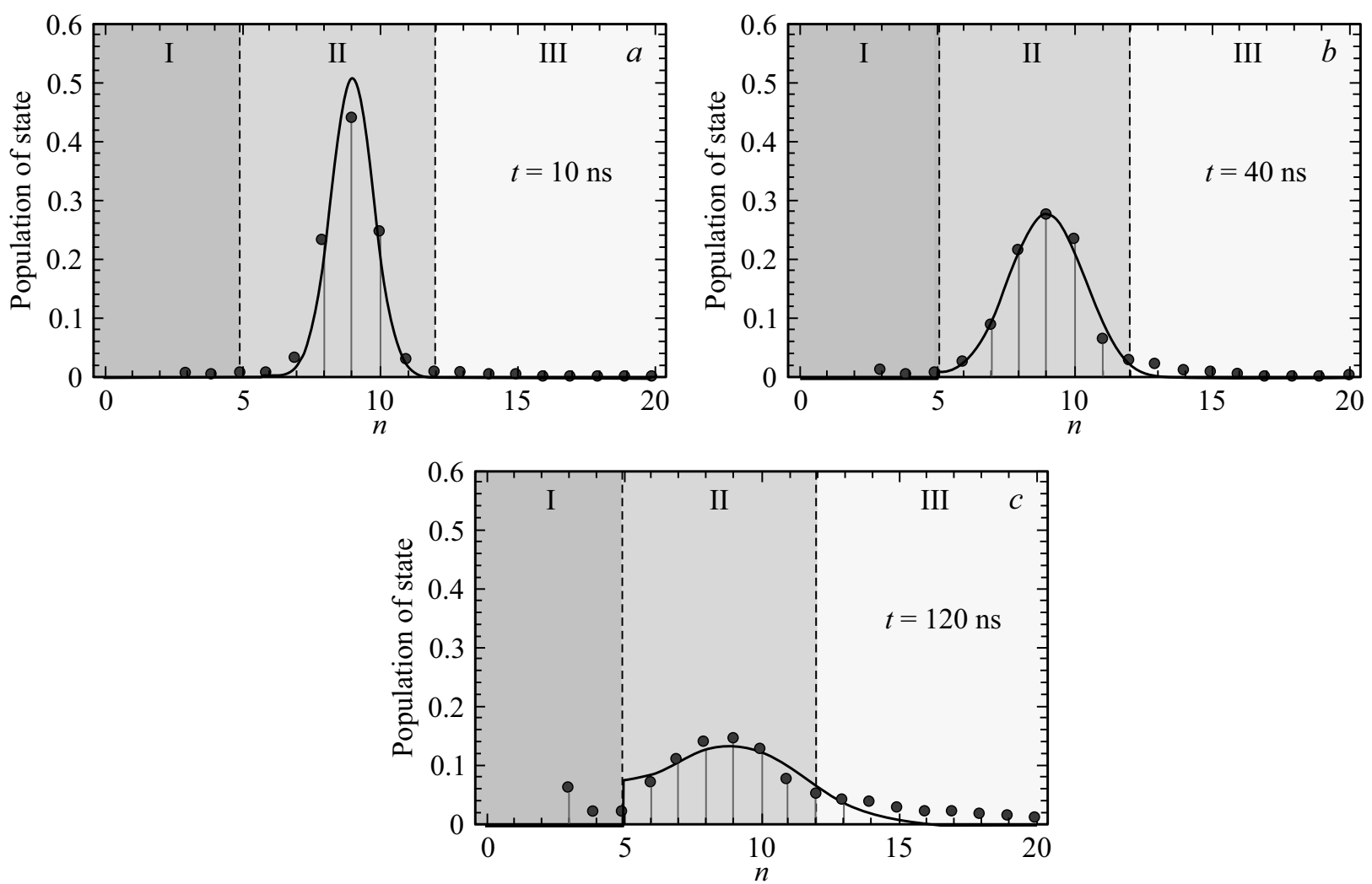

Рис. 5. Перераспределение заселенности состояний в атоме Na. Начальное состояние и параметры излучения такие же как на рис. 2. Точки - численное решение кинетических уравнений (1); сплошная - линия - диффузионное приближение (3)-(10).

Проинтегрировав уравнение (7) по $n$ в диапазоне от $n_{2}$ до $\infty$, получим уравнение для $P$ :

$$
\frac{d P}{d t}=-\left.D \frac{\partial N_{\mathrm{II}}}{\partial n}\right|_{n=n_{2}}-\left(w_{i}+w_{q}\right) P
$$

Решив уравнение (11), получим:

$$
\Omega_{i}(t)=-\left.w_{i} D \int_{0}^{t} \frac{\partial N_{\mathrm{II}}}{\partial n}\right|_{n=n_{2}} e^{\left(w_{i}+w_{q}\right)(\tau-t)} d \tau,
$$

где $\left.\frac{\partial N_{\text {II }}}{\partial n}\right|_{n=n_{2}}$ определяется выражением (10).

Таким образом, в рамках диффузионного приближения оказалось возможным получить явные выражения для заселенности состояний (9) и скорости ионизации (12).

На рис. 6 представлена скорость ионизации, рассчитанная по формуле (12) и полученная численно из (1). Можно видеть, что в начальный момент она равна 0 , потому что заселенность сосредоточена в области II, где прямая фотоионизация невозможна. Затем заселенность частично переходит в область III, соответственно скорость ионизации начинает расти, достигая своего максимального значения в момент времени

$$
t_{i} \approx 0.2\left(\frac{n_{2}-n_{0}}{\omega_{q} \sqrt{D}}\right)^{2 / 3}
$$

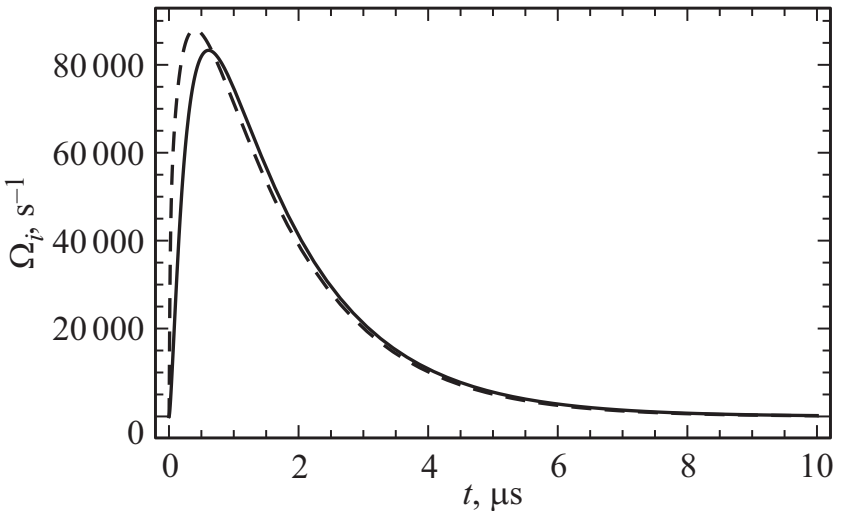

Рис. 6. Скорость ионизации атома $\mathrm{Na}$ (скорость уменьшения заселенности связанных состояний) в зависимости от времени. Начальное состояние и параметры излучения такие же как на рис. 2. Сплошная линия - численное решение кинетических уравнений (1); штриховая линия - расчет по формуле (12).

и далее медленно убывает, потому что суммарная заселенность состояний в области II и III уменьшается.

Представляет интерес оценить, какова должна быть минимальная спектральная плотность излучения, при которой существенная доля заселенности начального состояния уходит в непрерывный спектр, а не возвращается в основное состояние. Для этого, очевидно, 
время жизни состояния $\tau \sim 1 / w_{q}$ должно превышать характерное время ионизации $t_{i}$. Запишем коэффициент диффузии в виде $D \sim \rho C$, где $C-$ коэффициент пропорциональности, зависящий от $\omega_{\min }$ и $\omega_{\max }$. Тогда из (13) следует, что минимальная плотность излучения, при которой диффузионная ионизация становится существенной, дается выражением

$$
\rho_{\min } \approx 0.008 \frac{w_{q}\left(n_{2}-n_{0}\right)^{2}}{C} .
$$

Для ионизации атома $\mathrm{Na}$ из начального состояния $9 s$ под действием излучения с граничными частотами $\omega_{\min }=7 \cdot 10^{-4}, \omega_{\max }=5 \cdot 10^{-3}$ выражение $(14)$ дает $\rho_{\min } \approx 2 \cdot 10^{-16}$.

Выполненное рассмотрение остается справедливым и для излучения с непрямоугольным спектром. От профиля спектра будут зависеть лишь численные значения вероятностей переходов в кинетических уравнениях (1) и соответственно коэффициентов в уравнении ФоккераПланка (2) и в следующих из него приближенных решениях и оценках (9)-(14). Диффузионная динамика переходов под действием излучения с неограниченным спектром требует дополнительного анализа, но и для нее данное рассмотрение остается по большей части справедливым, если можно указать приближенные значения $\omega_{\min }$ и $\omega_{\max }$, понимая их как границы диапазона, в котором сосредоточена большая часть энергии излучения (например, в случае теплового излучения).

\section{3. Заключение}

Рассмотренный механизм диффузионной ионизации, кроме возможного применения для описания лабораторных экспериментов, может быть использован для анализа астрофизических данных. В частности, диффузионный механизм ионизации может вносить существенный вклад в вероятность ионизации атомов в верхних слоях хромосферы красных гигантов, где достаточно мала плотность частиц и соответственно маловероятна столкновительная ионизации.

Что касается рассмотренного в настоящей статье атома $\mathrm{Na}$, то актуальными являются многие вопросы, относящиеся к процессам в обогащенных натрием атмосферах коричневых карликов, а также спутника Юпитера Ио, где могут проявляться ионизационные процессы с участием возбужденного атома натрия [25].

\section{Финансирование работы}

Исследование выполнено при финансовой поддержке Российского фонда фундаментальных исследований (проект 19-32-90204) и Министерства науки и высшего образования РФ (проект FZGU-2020-0035).

\section{Конфликт интересов}

Авторы заявляют, что у них нет конфликта интересов.

\section{Список литературы}

[1] Bayfield J.E., Koch P.M. // Phys. Rev. Lett. 1974. V. 33. N 5. P. 258.

[2] Koch P.M., Van Leeuwen K.A.H. // Phys. Rep. 1995. V. 255. N 5-6. P. 289.

[3] Delone N.B., Zon B.A., Krainov V.P. // ZhETF. 1978. V. 75. P. 445.

[4] Jensen R.V. // Nature. 1992. V. 355. N 6358. P. 311.

[5] Casati G., Chirikov B.V., Shepelyansky D.L., Guarneri I. // Phys. Rev. Lett. 1986. V. 57. N 7. P. 823.

[6] Casati G., Ford J. // LNP. 1979. V. 93. P. 375.

[7] Galvez E.J., Sauer B.E., Moorman L., Koch P.M., Richards D. // Phys. Rev. Lett. 1988. V. 61. N 18. P. 2011.

[8] Delone N.B., Krainov B.P., Shepelyanskii D.L. // Phys. Uspekhi. 1983. V. 26. N 7. P. 551.

[9] Casati G., Chirikov B.V., Shepelyansky D.L., Guarneri I. // Phys. Rep. 1987. V. 154. N 2. P. 77.

[10] Kaulakis B.P. // JETP Lett. 1988. V. 47. N 6. P. 360.

[11] Burkhardt C.E., Corey R.L., Garver W.P., Leventhal J.J., Allegrini M., Moi L. // Phys. Rev. A. 1986. V. 34. N 1. P. 80.

[12] Ryabtsev I.I., Tretyakov D.B., Beterov I.I. // J. Phys. B. 2005. V. 38. N 2. P. S421.

[13] Beterov I.I., Tretyakov D.B., Ryabtsev I.I., Entin V.M., Ekers A., Bezuglov N.N. // New J. Phys. 2009. V. 11. N 1. P. 013052.

[14] Beterov I.I., Tretyakov D.B., Ryabtsev I.I., Ekers A., Bezuglov N.N. // Phys. Rev. A. 2007. V. 75. N 5. P. 052720.

[15] Bezuglov N.N., Borodin V.M., Kazanskii A.K., Klyucharev A.N., Matveev A.A., Orlovskii K.V. // Opt. Spectrosc. 2001. V. 91. N 1. P. 19.

[16] Kaulakys B., Ciziunas A. // J. Phys. B. 1987. V. 20. N 5. P. 1031.

[17] Bezuglov N.N., Borodin V.M., Eckers A., Klyucharev A.N. // Opt. Spectrosc. 2002. V. 93. N 5. P. 661.

[18] Давыдкин В.А., Зон Б.А. // Опт. и спектр. 1981. Т. 51. № 1. C. 25.

[19] Davydkin V.A., Ovsyannikov V.D., Zon B.A. // Las. Phys. 1993. V. 3. N 2. P. 449.

[20] Гореславский С.П., Делоне Н.Б., Крайнов В.П. // ЖЭТФ. 1982. T. 82. № 6. C. 1789.

[21] Delone N.B., Goreslavsky S.P., Krainov V.P. // J. Phys. B. 1989. V. 22. N 18. P. 2941.

[22] Delone N.B., Goreslavsky S.P., Krainov V.P. // J. Phys. B. 1994. V. 27. N 19. P. 4403.

[23] Schweizer W., Faßbinder P., Gonzalez-Ferez R. // At. Data Nucl. Data Tables. 1999. V. 72. N 1. P. 33.

[24] Лифиии Е.М., Питаевский Л.П. Физическая кинетика. М.: Наука, Глав. ред. физико-математической лит-ры, 1979. $533 \mathrm{c.}$

[25] Ключарев А., Безуглов Н. Элементарные процессы и ионизационные явления в газоразрядных средах. СПб.: Издательство СПбГУ, 2017. 244 с. 